\title{
LA «DIMENSIÓN RELIGIOSA» DE LA FENOMENOLOGÍA DE LA DONACIÓN DE J.-L. MARION
}

\author{
THE «RELIGIOUS DIMENSION» OF J.-L. \\ MARION'S PHENOMENOLOGY OF GIVENNESS
}

\author{
Jorge Luis RogGero \\ Universidad de Buenos Aires
}

RESUMEN: ¿Puede hablarse de un "giro teológico» respecto de la fenomenología de
ean-Luc Marion? ¿La acusación de Dominique Janicaud es pertinente? ¿Mantiene aún
alguna vigencia? Habría que responder que el diagnóstico de Janicaud es acertado y, al
mismo tiempo, que no lo es. Existe una «dimensión religiosa» en la fenomenología de
a donación, pero ésta no debe leerse como un obstáculo para la tarea fenomenológica.
Este artículo se propone establecer de qué modo opera esta «dimensión religiosa» en la
propuesta de Marion.

Palabras Clave: Martin Heidegger, Jean-Luc Marion, fenomenología, dimensión religiosa, giro teológico.

ABSTRACT: Can we speak about a «theological turn» in Jean-Luc Marion's phenomenology? Is Dominique Janicaud's accusation relevant? Does it still maintain any validity? We should respond that Janicaud's diagnosis is correct and at the same time, it is not. There is a "religious dimension» in the phenomenology of givenness, but this should not be read as an obstacle to the phenomenological task. This article aims to establish how this «religious dimension» operates in Marion's proposal.

\footnotetext{
* Instituto de Filosofía "Alejandro Korn», Facultad de Filosofía y Letras de la Universidad de Buenos Aires, Puán 480, piso 4to., CP 1406, Ciudad Autónoma de Buenos Aires. jorgeluisroggero@gmail.com
} 
KeYwords: Martin Heidegger, Jean-Luc Marion, phenomenology, religious dimension, theological turn.

\section{Introducción}

Hans-Georg Gadamer destaca la decisiva relevancia de la «dimensión religiosa» en Heidegger: los cuestionamientos medulares de su pensamiento responden a motivaciones religiosas $(1981,310)$. Por su parte, Dominique Janicaud señala una "dimensión teológica» en Marion, pero le adjudica un sentido negativo. Refiriéndose a la obra de Marion, Janicaud sostiene: «...su vacío fenomenológico sólo se explica por una doble referencia que todo lector informado conoce: la problemática de la superación de la ontología (o de la metafísica) y la dimensión propiamente teológica o espiritual» (1991, 101-102). ¿¿Puede sostenerse una «dimensión teológica» o religiosa ${ }^{1}$ con un sentido positivo en la fenomenología de la donación marioniana? ¿Qué conclusión puede extraerse respecto de la relación entre filosofía y teología en la obra de Marion luego del extenso recorrido que ésta ha llevado a cabo?

Una primera indicación la da el propio Marion en la serie de entrevistas realizadas por Dan Arbib, compiladas en La rigueur des choses. Allí el fenomenólogo francés responde de manera explícita a la acusación de Janicaud: «jamás hubo un "giro teológico de la fenomenología", ya que la fenomenología, desde un comienzo trató sobre temas teológicos [...] no se trata de un "giro", sino de un desarrollo y una extensión del campo de intervención de la fenomenología»

\footnotetext{
${ }^{1}$ Optaré por utilizar la expresión más amplia «dimensión religiosa» y no «dimensión teológica» por dos motivos: en primer lugar, para mantener la referencia a la religiöse Dimension propuesta por Gadamer; en segundo lugar, para distinguir esta «dimensión» de la propuesta teológica en sentido estricto, que puede encontrarse en algunos textos de Marion y que da cuenta, asimismo, de las reservas que el autor tiene con el término «teología». En Dieu sans l'être, Marion afirma: «La teología sólo puede acceder a su estatuto auténticamente teológico si se resuelve a deshacerse de toda teología. Si pretende hablar de Dios o más bien, de ese Dios (tachado) que tacha y borra todos los ídolos divinos, sensibles o conceptuales; si pretende así hablar de Dios (tachado), de manera que ese "de" se entienda tanto como el origen del discurso como su objetivo (no digo objeto, porque Dios (tachado) nunca puede servir de objeto y mucho menos en teología, a menos que se trate de una blasfemia), conforme al axioma que sólo "Dios habla bien de Dios"; y si, finalmente, ese Dios (tachado) estrictamente inconcebible, simultáneamente hablante y hablado, se da como el Verbo, como el Verbo dado hasta en la silenciosa inmediatez de la carne abandonada, entonces nada resulta más decente que la exposición de la lógica de esa teología al contragolpe, en ella misma, del theos» (1982, 197).
} 
(2012, 205). Y a continuación, en una suerte de argumento ad hominem, Marion descalifica el planteo de Janicaud por tratarse de un crítica hecha por alguien que no conoce bien la obra de Husserl. ${ }^{2}$ Apoyándose en el libro de Housset, Marion destaca como la ampliación de la fenomenología al dominio teológico fue una preocupación del propio Husserl. Como bien recuerda Housset, su filosofía estuvo interesada en la pregunta religiosa a tal punto que en una entrevista con Adelgundis Jaegerschmid, en 1935, Husserl sostuvo:

La vida del hombre no es otra cosa que un camino hacia Dios. Yo busco alcanzar esta meta sin el apoyo, las pruebas y el método de la teología; es decir, busco llegar a Dios sin Dios. Debo en cierto modo eliminar a Dios de mi vida científica para abrir a los hombres un camino hacia Dios, a aquellos que no tienen como Usted la seguridad de la fe a través la Iglesia. Sé que este procedimiento podría ser peligroso para mí si no estuviera profundamente unido a Dios y no fuera una persona que cree en Cristo $(1935,56)$.

En este sentido, Housset destaca que en Husserl la filosofía como ciencia rigurosa es también un camino hacia Dios y hacia una vida verdadera (2010, 18). Según Marion, esta preocupación religiosa es retomada por Heidegger y por Scheler, y es heredada por los fenomenólogos actuales. Sin embargo, ¿acaso esta respuesta diluye la pregunta por las fronteras entre filosofía (fenomenología) y teología? ¿Puede afirmarse sencillamente que esta cuestión ha perdido su pertinencia?

El estudio de Hans-Dieter Gondek y László Tengelyi, Neue Phänomenologie in Frankreich, publicado en 2011, permite atenuar la crítica de Janicaud, pues el concepto de "giro teológico" parece incapaz de dar cuenta de todo el arco que compone la fenomenología francesa actual. El libro ordena este campo heterogéneo en torno a la afirmación del "carácter acontecial» (Ereignisscharakter) de lo dado, que comparten todos los «nuevos fenomenológos» desde Richir hasta

\footnotetext{
2 «Pienso que estaba equivocado, y por una buena razón realmente simple: se había erigido en defensor de la ortodoxia husserliana, aunque él no era un gran conocedor de Husserl, sino un hegeliano y un heideggeriano cercano a Beaufret. Su lectura de Husserl era pues parcial, bastante superficial y en todo caso reciente» (Marion, 2012, 205).
} 
Marion $(2011,672)$. Sin embargo, sobre el final de la obra, Gondek y Tengelyi se preguntan por las temáticas «teológicas» que caracterizan la fenomenología francesa contemporánea y concluyen: «Quizás nuestro tiempo abre por primera vez un acceso fenomenológico a algunos temas fundamentales que durante milenios fueron reservados o acaparados por las tradiciones teológicas» $(2011,674)$. Los autores consideran que más que un «giro teológico», hay una utilización filósofica de temas teológicos que puede observarse en Henry, Marion y Chrétien, pero también en Barbaras o Richir $(2011,674) .^{3}$

Acordando con esta lectura de Gondek y Tengelyi, este trabajo sostiene como hipótesis que la fenomenología de la donación es delineada por Marion bajo un modelo teológico; lo cual, lejos de invalidar sus conclusiones filosóficas -como pretendería Janicaud-, las potencia. Siguiendo la estrategia del joven Heidegger, la apropiación de ciertas nociones teológicas llevada a cabo por Marion revitaliza su fenomenología considerada en términos estrictamente filosóficos.

A tal fin, en un primer apartado indagaré en el modo en que la «dimensión religiosa» opera en la "transformación hermenéutica» ${ }^{4}$ de la fenomenología llevada a cabo por el joven Heidegger. En un segundo apartado, a la luz del modelo heideggeriano, examinaré la manera en que la «dimensión religiosa» se manifiesta en la fenomenología de la donación. Finalmente, extraeré algunas conclusiones respecto de esta estrategia filosófica de apropiación de ideas teológicas y respecto del modo en que esta consideración afecta la relación misma entre filosofía y teología.

\section{La «dimensión religiosa» en la fenomenología hermenéutica del joven Heidegger}

En 1923, en el último curso dictado en la Universidad de Friburgo, antes de su partida a Marburgo, Heidegger sostiene: «Compañero en la búsqueda fue el joven Lutero y modelo Aristóteles, a quien aquél odiaba. Impulsos me dió Kierkegaard y los ojos me los colocó Husserl» $(1923,5)$. De este modo,

\footnotetext{
${ }^{3}$ A continuación, los autores proponen una clasificación de tres vías posibles de relación entre fenomenología y teología en estos autores: 1) «religión post-metafísica» (nachmetaphysischen Religion), ensayada por Marion, Chrétien, Henry, Ricœur y Depraz; 2) «cuasi-teología sin religión»(Quasi-Theologie ohne Religion), practicada por Derrida, Richir, Barbaras, Franck, Dastur y Escoubas; 3) «ateísmo no-metafísico» (nicht-metaphysischen Atheismus), propuesta por Benoist como una suerte de vía post-fenomenológica.

${ }^{4}$ Utilizo la expresión de Ramón Rodríguez (Rodríguez, 1997).
} 
Heidegger reconoce expresamente cuáles son las principales influencias en el desarrollo de su fenomenología. Entre ellas, no hay que subestimar la compañía del joven Lutero en la búsqueda de una radicalización hermenéutica de la fenomenología. En ese tiempo, indagando sobre su «yo soy», Heidegger llegó a definirse a sí mismo como un "teólogo cristiano» (christlicher Theologe), en una carta a Karl Löwith $(1921,29)$. Según Gadamer, la determinación del «yo soy», del sí mismo heideggeriano pasaba por esclarecer su cristianismo. Esta era la «motivación más profunda» (die tiefste Motivation) de su pensamiento y por esta razón Lutero "adquirió para él un significado decisivo» (1981, 310). El joven Heidegger de comienzos de la década del 20 buscaba -según le escuchó decir el propio Gadamer- «encontrar la palabra que es capaz de llamar a la fe y de hacer permanecer en la fe» $(1981,315)$. Esta preocupación heideggeriana forja la «dimensión religiosa» de su fenomenología temprana. En palabras de Gadamer: «...fue con propósitos teológicos y no bajo la influencia del historicismo que la historicidad de la existencia entró en su campo de visión y guió la pregunta por el sentido de "ser"» $(1981,315)$. La propuesta filosófica del joven Heidegger se encuentra atravesada por esta indagación teológica; sus principales categorías constituyen particulares apropiaciones de temas teológicos.

Como bien han destacado John van Buren (1994), Jaromir Brejdak (1996), Christian Sommer (2005), John McGrath (2006), Sylvain Camilleri (2008), entre otros, la theologia crucis o, mejor dicho, la tarea del teólogo de la cruz, ${ }^{5}$ constituye una suerte de modelo para la tarea del fenomenólogo heideggeriano. La heideggeriana hermenéutica de la facticidad se delinea según los rasgos de la teología del joven Lutero. Heidegger lee la theologia crucis como una teología de abandono fáctico de la creatura, una creatura que por su naturaleza no puede saber nada respecto de Dios (McGrath, 2006, 159; Brejdak, 1996, 180-182). En este sentido, la lectura heideggeriana de las cartas de Pablo presenta un "cristianismo de la finitud” -según la expresión de Marta Zaccagnini- un cristianismo

\footnotetext{
${ }^{5}$ Siguiendo la indicación de Jürgen Moltmann, cabe señalar que "Lutero no habla aquí de la teología como conocimiento de Dios en sí, sino del teólogo, es decir, del hombre que intenta conocer a Dios. Todo cristiano es para él 'teólogo', o sea, uno que conoce a Dios. ¿Qué hace de él el conocimiento de Dios? No se considera la teoría teológica en sí, su contenido y su método, sino la teoría en conexión con su usus por el hombre. A esto lo llamamos en el capítulo 2 paso de una teoría pura a una de cuño crítico, porque ya Lutero refleja aquí conocimiento e interés en sus contextos conscientes o inconscientes. Pregunta por el interés dominante en el conocimiento de Dios y por el uso del conocimiento por parte del hombre. Por eso no habla de una theologia gloriae, sino del theologus gloriae" (Moltmann, 1975, 290).
} 
"exclusivamente dirigido a la dimensión mundana de la experiencia de la vida y no a la dimensión ultraterrena de su trascendencia” $(2003,64)$.

Para dar cuenta de esta operación filosófica heideggeriana de apropiación de ideas teológicas me detendré en la ponencia sobre Lutero que el filósofo de Meßkirch expuso para el seminario de Rudolf Bultmann en 1924, en la Universidad de Marburgo. El texto da cuenta no sólo de la actitud fundamental de "humildad ante al misterio" de la revelación divina en la cruz, actitud que caracterizará la fenomenología del joven Heidegger como un detenerse ante el fenómeno para que éste se muestre en sus propios términos (van Buren, 1994, 319ss), sino que también es posible advertir como Heidegger toma nota de ciertos rasgos de la fenomenalización divina que serán trasladados a la dinámica de mostración del "fenómeno fundamental" (Grundphänomen) (1921-1922, 80): el fenómeno de la «vida fáctica» (faktisches Leben), y de la consecuente necesidad de implementar un método acorde a esta modalidad de aparición.

El texto comienza destacando la nueva dirección que Lutero imprime a la investigación teológica al erigir al problema del pecado como el insoslayable punto de partida. Todas las preguntas teológicas deben reconocer su dependencia respecto de esta cuestión. "Cuanto más se subestima la radicalidad del pecado, más se menosprecia la redención, y más pierde necesidad la encarnación divina» (Heidegger, 1924, 29). La concepción que se sostenga respecto del pecado será determinante respecto a la noción soteriológica y antropológica que se pretenda.

En la «Disputación de Heidelberg», Heidegger encuentra la más aguda formulación de la posición luterana respecto del pecado. Este querer ser Dios y no dejar a Dios ser Dios se manifiesta en una actitud teológica que vuelve al hombre soberbio. Esta actitud es la propia de la theologia gloriae escolástica que pretende decir «eso que la cosa es» (id quod res est) sin antes acudir a la cruz, es decir, que pretende determinar teóricamente el ser divino sin reparar en su manifestación sufriente en la cruz. Comenta Heidegger:

El escolástico toma conocimiento de Cristo sólo con posterioridad, después de que ha definido el ser de Dios y del mundo. Este enfoque griego del escolástico vuelve al hombre soberbio; él debe primero acudir a la cruz, antes de decir id quod res est $(1924,30)$. 
Pero, ¿en qué consiste este "acudir a la cruz»? Lutero propone una theologia crucis, una teología que no tema enfrentarse con el misterio de un Cristo crucificado. La cuestión principal la señala san Pablo en 1 Cor 1, 22-24:

Mientras los judíos piden milagros y los griegos van en busca de sabiduría, nosotros, en cambio, predicamos a un Cristo crucificado, escándalo para los judíos y locura para los paganos, pero fuerza y sabiduría de Dios para los que han sido llamados, tanto judíos como griegos. Porque la locura de Dios es más sabia que la sabiduría de los hombres, y la debilidad de Dios es más fuerte que la fortaleza de los hombres.

Dios se revela como Cristo crucificado. Dios no se manifiesta en términos de poder ni en términos de racionalidad. La cruz nos expone a la paradoja de la fe: el Cristo, Dios hecho hombre, muere del modo más cruel e infamante, reservado para los criminales. La propuesta luterana implica asumir la radicalidad de la fe cristiana, implica aceptar la dificultad de esta paradoja, de esta falta de respuestas, aceptar el estado de incertidumbre constante, «de absoluta tribulación (absolute Bedrängnis) que forma parte de la vida de los cristianos» (1920-21, 97). En la probatio de la tesis 20, Lutero sostiene que Cristo:

De esta manera «destruyó la sabiduría de los sabios», etc.; como dice Isaías: «Verdaderamente tú eres un Dios que te encubres». Así Juan 14, cuando Felipe decía, conforme a la teología de la gloria, «Muéstranos al Padre», Cristo pronto lo retrajo y reorientó su ilusorio pensamiento, el cual quería buscar a Dios en otra parte, diciéndole: «Felipe, el que me ve, ve también a mi Padre». Por consiguiente, en Cristo crucificado está la verdadera teología y el conocimiento de Dios $(1518,362)$.

La teología crucis toma como punto de partida al Deus absconditus en la cruz, a ese Dios que se encubre, que sólo se revela de manera indirecta y paradójica en 
el sufrimiento de Cristo crucificado. El Dios que se esconde en Cristo crucificado «destruye la sabiduría de los sabios» (1 Cor 1, 19) y nos invita a una actitud de humildad radical. La teología de la cruz parte de este carácter radical de la fe que debe entregarse al misterio que es escándalo y locura. Asumir la paradoja de la cruz e intentar dar cuenta de la misteriosa mostración de un Dios que se oculta, que se muestra bajo el modo del encubrimiento, es la difícil tarea que la teología debe emprender según Lutero.

La teología crucis se constituye en modelo para la fenomenología hermenéutica del joven Heidegger que como una suerte de "phaenomenologia crucis» también debe afrontar la mostración de un fenómeno (la vida fáctica) que se sustrae bajo la modalidad del enmascaramiento. En el curso del semestre de invierno de 1921-1922, Heidegger analiza las categorías del sentido de referencia (Bezugssinn) y de la movilidad (Bewegtheit) de la vida fáctica (1921-22, 100-155). En la dinámica de relucencia (Reluzenz) y preconstrucción (Praestruktion), el fenómeno de la vida se enmascara. La vida fáctica huye de sí misma, pero, nuevamente, en su estar "fuera de sí» (aus sich hinaus) también, de alguna manera, se enfrenta a sí misma, se dirige hacia sí misma bajo el modo del "fuera de sí» (1921-22, 123). La ruinancia (Ruinanz) es la figura última de esta movilidad, es la "especificidad fundamental de la movilidad de la vida» (Grundeigentümlichkeit der Bewegtheit des Lebens) que determina categorialmente el carácter de la movilidad de la relucencia y de la preconstrucción. Heidegger la indica formalmente como "la movilidad de la vida fáctica que "realiza" y "es" la vida fáctica en sí misma, como sí misma, por sí misma, a partir de sí misma, y en todo esto, contra sí misma» (1921-22, 131).

La ruinancia es inseparable de la vida fáctica pues es ella misma y, al mismo tiempo, se vuelve contra ella. La vida fáctica se caracteriza por una movilidad ruinante que se dirige hacia sí misma, es decir, hacia una constante caída que puede caracterizarse como la nada de la vida fáctica. Este movimiento de anonadamiento (Vernichtung) no debe ser entendido como una ausencia o vacío, sino como una forma de comparecencia de la vida fáctica bajo la modalidad de la «no presencia» (Nichtvorkommen). La vida se anuncia en forma opaca (undurchsichtig), como enigmática (rätselhaft) en su "no presencia» (1921-22, 147-148). Y son, precisamente, estas características las que dan lugar al cuestionamiento filosófico. Cuestionamiento que no es otra cosa que el movimiento contra-ruinante (gegenruinante Bewegtheit) que se da en la vida misma, generado por estos mismos rasgos (1921-22, 153). 
El fenómeno de la vida fáctica exhibe en su movilidad un tipo de mostración compleja que requiere una metodología acorde a esta complejidad. Heidegger advierte que la theología crucis de Lutero es una respuesta a una problemática similar. La idea de un Deus absconditus se erige en fenómeno modelo para la fenomenología. El Deus absconditus "se muestra como tal en su mostrarse» (Heidegger, 1923, 67), está ahí por sí mismo en la cruz, no representado ni considerado en modo indirecto. Y sin embargo, su manera de mostrarse es bajo la modalidad del encubrimiento. Para acceder a este fenómeno es necesario implementar algún tipo de estrategia interpretativa-destructiva. La destructio luterana, que designa tanto la tarea divina que «destruye las sabiduría de los sabios», como la tarea del teólogo de la cruz que debe colaborar con la divina destruyendo la falsas construcciones conceptuales de Dios formuladas por los teólogos de la gloria, ofrece el modelo de acceso para el fenómeno de la vida fáctica. «Hermenéutica es destrucción!» $(1923,105)$, dice Heidegger en su último curso en Friburgo, en clara alusión a Lutero. Heidegger propone una "hermenéutica de la facticidad», una hermenéutica de la vida fáctica que en un movimiento contra-ruinante permita explicitar el sentido propio de la vida fáctica, más allá de las tergiversaciones de su "estado de interpretada" (Ausgelegtheit) $(1923,15)$ en las que se encuentra. Se trata, pues, de despejar toda teorización que impida al fenómeno mostrarse en tanto tal. La filosofía, como la teología de la cruz, debe evitar la "soberbia» de intentar aprehender su objeto por medio de conceptualizaciones teóricas $a$ priori asumiendo una actitud de humilde entrega al acontecer del fenómeno en sus propios términos. Como el Deus absconditus, la vida fáctica también nos demanda no rehuir de la dificultad que implica la complejidad de su mostración. Por eso Heidegger afirma en estos cursos que la filosofía debe ser a-tea: «La filosofía debe en su radical cuestionamiento auto-impuesto ser a-tea [a-theistisch] por principio" (1921-22, 197). Pues ella, como la theologia crucis, también debe partir de la "ausencia de Dios» sin presuponer que ya lo ha alcanzado o definido. Su punto de partida también debe ser una actitud que no prejuzgue con construcciones conceptuales al fenómeno que intenta comprender. El ateísmo del joven Heidegger es un «ateísmo conceptual» (Fehér, 1996-97, 59) o un "ateísmo existencial” (Greisch, 2000, 217), un ateísmo que responde a la necesidad del cuestionamiento que es filosófica pero también religiosa, un ateísmo que asume la perplejidad e incertidumbre propia de nuestra existencia finita como el único punto de partida posible. 
Es en estas cuestiones decisivas de la búsqueda filosófica del joven Heidegger donde se advierte la «compañía» fundamental de Lutero.

\section{La «dimensión religiosa» en la fenomenología de la donación de Marion}

Como sucede con el joven Heidegger, la «dimensión religiosa» de la fenomenología marioniana no debe asignarse al mero abordaje de fenómenos o temática religiosas, sino en el modo en que ciertas ideas forjadas en el campo teológico son apropiadas y utilizadas para revitalizar el campo filosófico.

Marion sostiene en Étant donné una distinción estricta entre el campo de la teología y el de la filosofía. Reflexionando sobre el fenómeno de la revelación, afirma:

La fenomenología describe posibilidades y no considera nunca el fenómeno de la revelación más que como una posibilidad de la fenomenicidad, que formularía así: si Dios se manifiesta (o se manifestara), se valdrá de una paradoja de segundo grado; si tiene lugar la Revelación (de Dios por él mismo, teo-lógica), tomará la figura fenoménica del fenómeno de revelación, de la paradoja de paradojas, de la saturación de segundo grado. Ciertamente, la Revelación (como efectividad) no se confunde jamás con la revelación (como fenómeno posible), respetaremos escrupulosamente esta diferencia conceptual mediante su traducción gráfica. Pero la fenomenología, que le debe a la fenomenicidad el llegar hasta ese punto, no va más allá y no debe nunca pretender decidir del hecho de la Revelación, ni de su historicidad, ni de su efectividad, ni de su sentido. No debe hacerlo pues, no solamente para distinguir los saberes y delimitar las regiones respectivas, sino en primer lugar porque no tiene los medios: el hecho (si lo hay) de la Revelación excede el dominio de toda ciencia, incluida la fenomenología; sólo una teología, y a condición de dejarse construir a partir de ese sólo hecho (K. Barth o H. U. von Balthasar, sin duda en mayor medida que R. Bultmann o K. Rahner) podría eventualmente acceder a ella. Incluso si lo deseara (y, por supuesto, jamás fue el caso), la fenomenología no tendría el poder de efectuar un giro hacia la teología. Y hay que ignorarlo todo sobre teología, sobre sus 
procedimientos y sus problemáticas, para considerar tan siquiera esa inverosimilitud (1997, 329).

La fenomenología (y la filosofía) debe limitarse a analizar la posibilidad del fenómeno de la revelación, y será tarea de la teología indagar en su efectividad, la distinción disciplinaria debe sostenerse sin excepciones. En el \$7, en respuesta a la crítica de Janicaud, Marion afirma que la donación no depende de un esquema sobrecargado de sentido al provenir de un "préstamo indiscreto de la teología», porque a partir de Husserl la donación desempeña un rol de pleno derecho en fenomenología (1997, 104-105). La réplica de Marion es clara, pero esto no implica que no pueda existir una impronta teológica en ciertas nociones fundamentales de la fenomenología de la donación. Como bien destaca Emmanuel Falque, por momentos, la escrupulosa preocupación de Marion por distinguir la fenomenología de la teología lo lleva casi a una confesión de parte respecto de la acusación de Janicaud $(2007,182)$. ¿No sería conveniente asumir el "préstamo teológico» y, en todo caso, explicar sus alcances y el modo filosófico de su empleo?

A continuación, intentaré mostrar el modo en que Marion se apropia filosóficamente de ideas provenientes de la reflexión teológica deteniéndome en un artículo de 1989 publicado en la revista Communio, intitulado «À Dieu, rien d'impossible». En este texto, previo a la publicación de Étant donné y del artículo «Le phénomène saturé», Marion analiza la noción de «milagro». Examinando la paradoja de la Resurrección, como milagro paradigmático, el fenomenólogo francés propone caracterizarla como un "fenómeno saturante» (phénomène saturant). ${ }^{6}$

\footnotetext{
${ }^{6}$ En Étant donné, Marion aclara por qué modificó la expresión «fenómeno saturante» por «fenómeno saturado». «Proponemos hablar de fenómeno saturado y no saturante, como a veces nos lo han sugerido. En efecto, es la intuición la que satura todo concepto o significación, de modo que ese fenómeno se manifiesta bajo un modo saturado por intuición saturante. Es más, la intuición que lo satura lo satura únicamente en nombre de la donación: el fenómeno saturado está, de entrada, saturado de donación. Un tal fenómeno, en efecto, satura posteriormente y en consecuencia la mirada a la que se da a ver y conocer; podríamos llamarlo también, si se quiere, saturante. Sin embargo, la saturación que ejerce en el campo del conocimiento deriva solamente de la que recibe en el campo de la donación, la donación determina siempre el conocimiento y no al revés» $(1997,276)$.
} 
El texto comienza analizando el uso de esta categoría en la cotidianidad. En este ámbito, Marion destaca que llamamos «milagro» «a cualquier acontecimiento cuya efectividad prueba la posibilidad de lo que antes yo consideraba definitivamente imposible» $(1989,45)$. De esta definición se siguen tres consecuencias: 1) En estos casos, la efectividad no sucede a la posibilidad sino que, por el contrario, la efectividad precede a la posibilidad. 2) El milagro demuestra que al menos en esta ocasión, lo posible ocurrido efectivamente superó lo posible previsto por mí, y esto debe llevarnos a reflexionar que no se trata de oponer la racionalidad a la credulidad, sino de aceptar o no los hechos que se nos imponen a pesar nuestro. 3) El milagro no pone en cuestión las «leyes de la naturaleza», sino la amplitud de lo posible.

A continuación, el texto evalúa el sentido teológico del «milagro». Como si intuyera la operación que llevará a cabo en textos posteriores forjando la categoría filosófica de "fenómeno saturado", Marion comienza este apartado del artículo sosteniendo: «El concepto de milagro, por tanto, no pertenece al dominio estrictamente religioso, sino que depende del análisis conceptual, si no propiamente filosófico, de la cotidianidad» $(1989,46)$. El milagro da cuenta de un modo de manifestación que excede el ámbito religioso.

El apartado continúa esbozando las características propias de los milagros de Cristo y se detiene en el «mayor milagro», en el milagro de la Resurrección, que no es hecho por Cristo, sino recibido del Padre por el Espíritu. La Resurrección revela la paradoja de la "posibilidad de la imposibilidad», invierte «la entropía del pecado al someter la muerte a la vida y ya no la vida a la muerte» (1989, 48). Ante la Resurrección, y en este sentido es el milagro paradigmático, 1) en primer lugar, nos vemos obligados a decidir si la aceptamos o la rechazamos como muerte de la muerte, pues nos urge nuestra propia muerte. No se trata de creer o no en una efectividad de lo imprevisible, desde un punto de vista teórico o contemplativo. El milagro de la Resurrección nos involucra a nivel existencial, y nos invita a un "trabajo crítico» (travail critique) en el que descubrimos que la posibilidad es más rica y amplia de lo pensado. De este modo, se accede también a «una racionalidad más comprensiva» $(1989,50)$. 2) En segundo lugar, el milagro de la Resurrección nos exige una decisión sin demora, pues «jamás las informaciones complementarias (pues de éstas ha habido después de dos mil años de reflexión) podrán colmar la distancia entre el acontecimiento y su inteligibilidad completa». La Resurrección nos coloca no ya ante un «acontecimiento de la posibilidad», sino ante "la posibilidad misma que se hace acontecimiento en el seno de la imposibilidad» $(1989,50)$. 
En el tercer apartado, Marion advierte que la inversión de la primacía de la efectividad por sobre la posibilidad puede ser rastreada en la historia de la metafísica. Nuevamente, anticipando el uso filosófico del concepto de milagro, Marion indaga en el campo mismo de la filosofía. La historia de la metafísica, desde Aristóteles en adelante, es la historia del sometimiento de la posibilidad a la efectividad. Aristóteles sostiene: «es evidente que el acto (enérgeia) es anterior a la potencia (dýnamis)» (Metafísica, IX, 1049b5). Este dictum ha fijado el tipo de relación entre posibilidad y efectividad en la historia de la metafísica. La fórmula kantiana "condiciones de posibilidad» indica que la posibilidad admite condiciones. Esto implica que la posibilidad «no se impone como posibilidad a partir de sí misma, sino que se deja definir: la posibilidad, también y sobre todo, obedece a las condiciones de su propia posibilidad» (Marion, 1989, 51). Las dos principales condiciones que se imponen a la posibilidad son los principios metafísicos de 1) contradicción y 2) de razón suficiente. El milagro desafía la idea de que 1) «nada contradictorio es posible (ni verdadero)» y que 2) «nada de lo que ocurre sin causa es verdadero (ni, por lo tanto, verdaderamente posible)» $(1989,51)$.

Sin embargo, no hay que abandonar el campo de la filosofía para encontrar la inversión del principio aristotélico. Según Marion, es la fenomenología la encargada de cuestionar la autoridad del Estagirita. En primer lugar, el principio de todos los principios, formulado por Husserl en el $\$ 24$ de Ideas $I$, afirma que la cuestión de derecho no proviene de una instancia externa a los fenómenos, sino que depende de una donación a la intuición. Los fenómenos ya no deben justificarse ante el Tribunal de la Razón, sino que se trata de dar crédito a lo que aparece. De este modo, se libera la posibilidad. Pues, como bien destaca Heidegger, «más elevada que la efectividad se encuentra la posibilidad» (1927, 51-52).

En este punto, Marion explicita su estrategia de apropiación filosófica de conceptos teológicos al preguntarse:

¿Cómo puede el milagro inscribirse en la perspectiva fenomenológica? En esto primeramente, en que la fenomenología libera la posibilidad al renunciar a deducirla de cualquier condición previa y al decidir después de la donación (Marion, 1989, 53). 
El «milagro» se inscribe en la perspectiva fenomenológica como «fenómeno saturado», pues el «milagro ofrece el ejemplo más puro de donación fenomenológica» $(1989,54)$. Sin embargo, en esta instancia, todavía no es posible dar este paso, pues la fenomenología todavía responde a los límites del horizonte y del Yo constituyente.

En el apartado final, titulado "La paradoja», Marion delinea las características decisivas del fenómeno saturado a partir de un análisis del milagro paradigmático de la Resurrección en relación al horizonte y al Yo constituyente. Marion destaca que el marco del horizonte, si bien conviene a todo milagro que pertenezca a este mundo, no es adecuado para un milagro que remita al exterior del mundo. La Resurrección es aquel fenómeno que aparece rechazando los límites de cualquier horizonte. Pero, ¿cómo es esto posible? ¿Cómo puede un fenómeno que rechaza todo horizonte aparecer en el horizonte del mundo? Marion responde introduciendo la categoría de "fenómeno saturado» o, mejor dicho, de «fenómeno saturante».

Por fenómeno saturante entendemos aquel en el que lo dado manifiesto sobrepasa no sólo lo que la mirada humana puede soportar sin cegarse y morir, sino lo que el mundo en su finitud puede recibir y contener [...] aquí [...] la donación intuitiva sobrepasa infinitamente lo que nuestra mención intencional puede esperar de significaciones y de esencias, como también lo que nuestra intuición puede soportar de plenitud $(1989,56)$.

Marion destaca que el error común que se comete al caracterizar los milagros es adjudicarles una penuria de intuición, de donación y de manifestación, que debería ser compensada por la fe por medio de una "apuesta» (pari) (1989, 56). Lo que sucede, según Marion, es precisamente lo contrario, lejos de darse defectuosamente, el milagro se da en exceso, saturando nuestra capacidad de recepción.

Respecto del Yo constituyente, anticipando nuevamente una característica definitoria del «fenómeno saturado", Marion sostiene que la Resurrección, en tanto fenómeno saturante, no se deja constituir, sino que «aqui el fenómeno constituye al Yo» $(1989,57)$. Y al reconocerse constituido, el Yo trascendental 
deviene un yo interpelado que debe responder a la donación en lugar de objetivarla. De este modo, Marion prefigura al adonado.

Este artículo clave en el desarrollo del itinerario del pensamiento marioniano permite dar cuenta de cómo la reflexión teológica ha sido el cantero del cual Marion extrajo ideas fundamentales para su filosofía.

\section{Conclusión}

Marion y Heidegger comparten una actitud similar: después de haber demostrado un profundo interés por las problemáticas teológicas, en pos de legitimar su trabajo filosófico, han buscado establecer una frontera férrea entre los dos saberes. El Heidegger posterior a Ser y Tiempo, luego de haber nutrido su pensamiento temprano con ideas teológicas, niega toda posibilidad de articulación entre la teología y el pensar en sentido propio. En Introducción a la metafisica, Heidegger descarta la posibilidad de un entrecruzamiento productivo entre filosofía y teología: «una filosofía cristiana es un "hierro de madera" [hölzernes Eisen] y un malentendido" (1935, 9). En Nietzsche II se encuentra esta misma idea: «Una "filosofía cristiana” es un contrasentido aún mayor que la idea de un círculo cuadrado [viereckige Kreis]» (1939-46, 132). Sin llegar al extremo de estas declaraciones heideggerianas, al insistir de modo recurrente en la necesidad de distinguir con claridad entre filosofía y teología, Marion tampoco parece estar dispuesto a reconocer la productividad del diálogo entre ambas disciplinas presente en su pensamiento.

Janicaud señala como problemática la «traducción» de la preocupación teológica en el campo de la fenomenología (1991, 85). Janicaud impugna la posibilidad de una inspiración religiosa de ciertas ideas filosóficas, lo que, por cierto, implicaría impugnar buena parte de la historia de la filosofía. Basta con recordar el modelo trinitario utilizado por Hegel. Como bien destaca Emmanuel Falque, hay una unidad en la obra de Marion que puede pensarse a partir de la idea de larvatus pro Deo, en el sentido de un avanzar enmascarado frente al «deslumbramiento ante la gloria divina» (2007, 185-186). Es ese deslumbramiento teológico el que guía también a sus innovaciones en el ámbito filosófico. Y esto no implica restarle validez ni importancia a su trabajo filosófico en tanto tal. En palabras de Romano: «Sería grosero e ingenuo pensar que todas las paradojas no son aquí más que una expresión de la Paradoja, con "p" mayúscula. Esta filosofía no es una teología disfrazada» $(2015,27)$. Por el contrario, la obra de Marion da 
cuenta de un movimiento que caracteriza a muchos autores contemporáneos, un gesto que bien puede caracterizarse como propio de un "giro teológico", y que implica la revisión de la secularización, la reapertura del diálogo entre filosofía y teología. Marion, así como Lévinas, Chrétien o Falque, pero también Agamben, Derrida o Nancy, en la huella de Kierkegaard, Heidegger y Benjamin, no buscan imponer una "teología disfrazada», sino encontrar en la teología nociones que permitan revitalizar la filosofía. ¿Es acaso esta operación inválida? ¿Es la frontera entre teología y filosofía un límite inquebrantable? Son elocuentes las palabras de Chrétien:

No basta instalarse como aduanero en la frontera supuestamente segura entre la filosofía y la teología; primero es preciso interrogarse como filósofo, sobre el propio trazado de esa frontera $(1992,10){ }^{7}$

En 2008, en el «Prólogo» a la edición española de Étant donné, interrogándose sobre el trazado de la frontera, Marion sostiene que para comprender la novedad del fenómeno saturado que aparece sin o contra toda constitución de objeto, es necesario ya no tomar a la frontera entre filosofía y teología como un "dogma incuestionable», sino que hay que pensar tal frontera como el producto de la historia de la metafísica $(2008,13)$. En una entrevista de 2014, retomando esta idea, Marion afirma que el problema de la distinción entre filosofía y teología es un problema metafísico pues implica suponer que la filosofía puede fijar las condiciones de la verdad en tanto «Tribunal».

Ahora bien, encontramos que, de hecho, casi ningún filósofo contemporáneo pretende aún [...] fijar las condiciones de posibilidad de la experiencia a priori. En consecuencia, la oposición, ya sea conflictiva o con ánimo conciliador, entre filosofía y teología aparece de ahora en más como la oposición en un partido amistoso $(2014,72)$.

${ }^{7}$ El propio Marion cita esta palabras de Chrétien en De surcroît (2001, 32 n. 2). 
Marion parece haber revisado la posición de su obra de 1997, probablemente porque ha advertido el potencial filosófico de la "dimensión religiosa» de su filosofía. No se trata de desconocer toda diferencia entre filosofía y teología. Está claro que para Marion aún es posible distinguir dos campos disciplinarios. Sin embargo, en nuestro tiempo postmetafísico devienen fundamentales dos cuestiones. En primer lugar, conviene preguntarse filosóficamente si el trazado de la frontera entre filosofía y teología debe continuar haciéndose desde los cánones de la metafísica. En segundo lugar, conviene advertir filosóficamente la riqueza que el diálogo con la teología provee al pensamiento filosófico.

\section{Agradecimientos}

Agradezco especialmente al Prof. Dr. Roberto Walton por su invaluable guía en mis investigaciones. 


\section{Bibliografía}

Aristóteles (I982). Metafísica, trad. T. Calvo Martínez. Madrid: Gredos.

brejdak, J. (I 996). Philosophia Crucis. Heideggers Beschäftigung mit dem Apostel Paulus. Frankfurt am Main: Peter Lang Verlag.

Camilleri, S. (2008). Phénoménologie de la religion et herméneutique théologique dans la pensée du jeune Heidegger. Commentaire analytique des Fondaments philosophiques de la mystique médiévale (1916-1919). Dordrecht: Springer.

Caputo, J. D. (1999). “Towards a postmodern Theology of the Cross. Augustine, Heidegger, Derrida”. Ed. Westphal, M. Postmodern Philosophy and Christian Thought. Bloomington: Indiana University Press.

Chrétien, J.-L. (1992). L’appel et la réponse. Paris: Éditions de Minuit.

FAlque, Emmanuel (2007). «Larvatus pro Deo. Jean-Luc Marion’s Phenomenology and Theology». Ed. Hart, K. Counter-experiences. Reading Jean-Luc Marion. Notre Dame: University of Notre Dame Press.

FEHÉr, I. M. (I996-97). «Heidegger's understanding of the atheism of philosophy: philosophy, theology, and religion on his way to Being and Time». Existentia, Vol. VIVII, Fasc. 1-4, pp. 33-64.

Gadamer, H.-G. (1981). "Die religiöse Dimension». Gesammelte Werke. Band 3: Neuere Philosophie: Hegel, Husserl, Heidegger. Tübingen: J. C. B. Mohr Verlag, 1987.

Gondek, H.-D. / Tengelyi, L. (2011). Neue Phänomenologie in Frankreich. Berlin: Suhrkamp.

Greisch, J. (2000). L'arbre de vie et l'arbre du savoir. Le chemin phénoménologique de l'herméneutique heideggérienne (1919-1923). Paris: Cerf.

Heidegger, M. (1920-21). «Einleitung in die Phänomenologie der Religion». Gesamtausgabe. II. Abteilung: Vorlesungen 1919-1944. Band 60: Phänomenologie des religiösen Lebens. Frankfurt am Main: Vittorio Klostermann, 1995.

-(I92 I). «Drei Briefe Martin Heidegger an Karl Löwith». Hrsg. Papenfuss, D. / Pöggeler, O. Zur philosophischen Aktualität Heideggers. Band 2: Im Gespräch der Zeit. Frankfurt am Main: Vittorio Klostermann, 1990.

-(I92 I-22). Gesamtausgabe. II. Abteilung: Vorlesungen 1919-1944. Band 61: Phänomenologische Interpretationen zu Aristoteles. Einführung in die phänomenologische Forschung. Frankfurt am Main: Vittorio Klostermann, 1985.

-(1923). Gesamtausgabe. II. Abteilung: Vorlesungen 1919-1944. Band 63: Ontologie. Hermeneutik der Faktizität. Frankfurt am Main: Vittorio Klostermann, 1988. 
-(1924). «Das Problem der Sünde bei Luther». Hrsg. Jaspert, B. Sachgemäbe Exegese: Die Protokolle aus Rudolf Bultmanns Neutestamentlichen Seminaren I92 I-I95I, Marburg, N. G. Elwert Verlag, 1996.

-(1927). Gesamtausgabe. I. Abteilung: Veröffentlichte Schriften 1910-1976. Band 2: Sein und Zeit. Frankfurt am Main: Vittorio Klostermann, 1977.

-(1935). Gesamtausgabe. II. Abteilung: Vorlesungen 1919-1944. Band 40. Einführung in die Metaphysik. Frankfurt am Main: Vittorio Klostermann, 1983.

-(1939-46). Gesamtausgabe. I. Abteilung: Veröffentlichte Schriften 1910-1976. Band 6.2: Nietzsche II. Frankfurt am Main: Vitttorio Klostermann, 1997.

Housset, E. (2010). Husserl et l'idée de Dieu. Paris: Cerf.

Husserl, Edmund (1935). «Gespräche mit Edmund Husserl, 1931-1936». Jaegerschmid, A. OSB. Stimmen in Zeit, Heft 1 (Januar 1981), pp. 48-58.

JANICAUD, D. (1991). «Le tournant théologique de la phénoménologie française». La phénoménologie dans tous ses états. Paris: Gallimard, 2009.

Luther, M. (1518). D. Martin Luthers Werke. Kritische Gesamtausgabe, Band 1: Schriften, Predigten, Disputationen 1512/18, Weimar, Hermann Böhlau Nachfolger, 1883.

Marion, J.-L. (1982). Dieu sans l'être. Paris: PUF, 1991.

-(1989). «À Dieu, rien d'impossible». Communio. Revue Catholique Internationale, XV, 5, pp. 43-58.

-(1997). Étant donné. Essai d'une phénoménologie de la donation. Paris: PUF.

-(2001). De Surcroît. Études surles phénomènes saturés. Paris: PUF.

-(2008). «Prólogo a la edición española». Siendo dado. Ensayo para una fenomenología de la donación, trad. J. Bassas Vila. Madrid: Síntesis.

-(20 12). La rigueur des choses. Entretiens avec Dan Arbib. Paris: Flammarion.

-(2014). «Foi et raison. Jean-Luc Marion et Laurence Devillairs». Études, 2, pp. 67-76.

McGrath, Sean J. (2006). The early Heidegger and medieval philosophy: phenomenology for the Godforsaken. Washington: The Catholic University of America Press.

Moltmann, Jürgen (1975). El Dios crucificado. La cruz de Cristo como base y critica de toda teología cristiana, trad. S. Talavero Tovar. Salamanca: Sígueme.

RodRíGUEZ, R. (1997). La transformación hermenéutica de la fenomenología. Una interpretación de la obra temprana de Heidegger. Madrid: Tecnos.

Romano, C. (2015). «Le don, la donation et le paradoxe». Éd. Capelle-Dumont, Ph. Philosophie de Jean-Luc Marion. Paris: Hermann. 
Sommer, Ch. (2005). Heidegger, Aristote, Luther. Les sources aristotéliciennes et néo-testamentaires $d$ Être et temps. Paris: PUF.

van Buren, J. (1994). The young Heidegger. Rumor of the hidden king. Bloomington: Indiana University Press.

ZaCCagnini, Marta (2003). Christentum der Endlichkeit. Heideggers Vorlesungen: Einleitung in die Phänomenologie der Religion. Münster: LIT Verlag.

Recibido : 27/07/2016

Aceptado : 26/06/2017

\section{(c) $(1) 99$}

ENDOXA está bajo una licencia de Creative Commons Reconocimiento-NoComercial-SinObraDerivada 4.0 Internacional 